\title{
COMPORTAMENTO ORGANIZACIONAL: UMA APLICAÇÃO DA TIPOLOGIA DE MILES E SNOW NO SETOR HOTELEIRO DE FLORIANÓPLIS, SC
}

\author{
ORGANIZATIONAL BEHAVIOR - AN APPLICATION OF THE \\ TYPOLOGY OF MILES AND SNOW IN THE HOSPITALITY \\ INDUSTRY FROM FLORIANOPOLIS - SC
}

\author{
Recebido - 15/03/2011 \\ Aceito - 05/08/2011 \\ Marison Luiz Soares ${ }^{1}$, Omar Raimundo De Paula Teixeira ${ }^{2}$ \\ e Anderson Soncini Pelissari ${ }^{3}$
}

\begin{abstract}
Resumo
Este estudo teve como objetivo verificar o comportamento estratégico adotado pelos administradores de hotéis de Florianópolis, SC, segundo a tipologia de Miles e Snow (1978). Optou-se pela abordagem quantitativa e descritiva, utilizando-se, para coleta dos dados, questionários fechados. Os resultados demonstraram que $45 \%$ dos administradores de hotéis de Florianópolis adotam comportamentos prospectores; $31 \%$, analistas; $19 \%$, reativos e $5 \%$, defensivos. Conclui-se, portanto, que os administradores adotam, em sua maioria, comportamentos prospectores, que se caracterizam pela busca de novos mercados e introdução de novos produtos e serviços aos clientes, salientando-se que, de um modo geral, foram preponderantes os comportamentos prospectores e analistas que, somados, perfizeram um percentual de $77 \%$ dos comportamentos identificados.
\end{abstract}

Palavras-chave: Setor hoteleiro. Comportamento. Estratégia organizacional.

\section{Abstract}

The study was to verify the strategic behavior adopted by administrators hotels Florianópolis-SC, Brazil, according to the typology of Miles and Snow (1978). The search is characterized as a quantitative, survey-type, it has a population of 22 hotels located in Florianopolis-SC, Brazil, falling between three and five stars. We opted for the quantitative and descriptive approach, using for data collection, closed questions. The results showed that $45 \%$ of Florianópolis hotels administrators adopt behaviors Surveyors, 31\% Analysts, 19\% Reactives and Defensives 5\%. The conclusion is that administrators adopt mostly Prospectors behaviors that are characterized by the pursuit of new markets and introduction of new products and services to customers.

Keywords: Strategic Behavior. Organizational Behavior. Typology of Miles and Snow. Organizational strategy and Hospitality Industry

\footnotetext{
1 Mestre em Administração - Professor da UNITINS - marison@matrix.com.br

2 Doutor em Engenharia da Produção - Professor Adjunto da Universidade Federal do Espírito Santo - UFES omar.rp@unitins.br

3 Doutor em Engenharia de Produção pela Universidade Metodista de Piracicaba - UNIMEP/SP. Professor do Departamento de Administração da UFES (Universidade Federal do Espírito Santo) - asoncinipelissari@gmail.com.
} 


\section{INTRODUÇÃO}

As organizações têm apresentado, nos últimos tempos, significativas transformações em seu comportamento e, ao contrário da administração clássica, que considera as organizações como um sistema fechado, sendo que tudo é concebido como funcional e previsível, a teoria organizacional contemporânea assume uma perspectiva bem mais ampla das organizações, que são vistas como sistemas abertos.

Ao examinarem a relação entre estratégia e comportamentos gerenciais, Gupta e Govindarajan (1982) verificaram que as mudanças sugerem novas formas de organizar e conduzir as empresas, seja em termos de estratégia ou gestão, seja na administração com maior propensão ao risco e tolerância à ambiguidade por aqueles que estavam fortemente associados às estratégias e também eram os que tinham uma melhor performance.

Dill (1958) foi um dos primeiros a estudar o comportamento organizacional e dar importância às influências do ambiente sobre a ação gerencial. Posteriormente, outros trabalhos também trataram desse assunto e, mesmo com enfoques diferentes, todos são unânimes em reconhecer sua importância.

Chandler (1962), Emery e Trist (1965), Duncan (1972), Lawrence e Lorch (1973) e Katz e Kahn (1987) trabalharam com o enfoque de que o comportamento das organizações é determinado pelas ações do meio em que atuam. Dentre os que entendem que as organizações são selecionadas por serem mais aptas, destaca-se o trabalho de Hannan e Freeman (1977). Nos estudos em que se preconiza que os administradores adotam comportamentos e buscam entender seu ambiente e tomar decisões estratégicas que visam obter os recursos de que necessitam, foram notórios os trabalhos de Child (1972) e Pfeffer e Salancik (1978). Outros autores, como Zucker (1987) e Powel e DiMaggio (1991) entendem que as pressões fazem com que, com o tempo, o comportamento das organizações, em um determinado ambiente, pareça igual. Dentre os trabalhos que evidenciam que as organizações devem buscar modelos de comportamento que as permitam garantir a congruência entre a estrutura, a forma de condução e os fatores contingenciais internos e externos, encontram-se o de Miles e Snow (1978), Milliken (1987), Miller e Dess (1993) e Mintzberg (1995).

Katz e Kahn (1987), ao estudarem o comportamento organizacional, salientaram que a sua dinâmica precisa ser analisada sob a ótica das transações contínuas com o meio que o ampara, porque é a partir das interpretações dos fenômenos ambientais que a organização deverá adotar posturas de adaptação estratégica.

Segundo Machado-da-Silva e Fonseca (1993), a relação entre organização e estratégia seria mediada por fatores que afetariam a percepção, a interpretação e o comportamento dos agentes organizacionais em relação às pressões contextuais. Nesta mesma linha, Rossetto (1998) estudou o processo de adaptação estratégica de três empresas do setor da construção civil e percebeu que o comportamento das organizações está relacionado com a percepção que seus gestores têm do ambiente no qual atuam.

Para Mintzberg, Ahlstrand e Lampel (2000), o estudo do comportamento das organizações é importante, como também é preferível concentrar-se no ambiente e ter uma leitura, mesmo que aproximada, dos fatos a ter que se submeter às suas incertezas, pois, segundo Cusumano e Markides (2002), é difícil reverter as grandes decisões, porque elas envolvem um sério comprometimento de capital e de pessoal.

Se o momento é de incertezas, então as atenções para a orientação estratégica devem ser prioridades da administração (JABNOUN; KHALIFAH; YUSUF, 2003). Percebe-se, portanto, 
que independentemente do tipo de comportamento adotado pelas organizações, elas estarão sempre diante de novas situações, proporcionadas pelos ambientes e seus agentes.

Tan e Tan (2005) investigaram, por 12 anos, a adaptação estratégica em organizações chinesas, concluindo que os comportamentos são moderados por estágios durante a transição pela qual as organizações passam e que especificamente as firmas mais jovens são mais proativas que as organizações mais antigas.

Por sua vez, Frishammar (2006) salienta que muitos livros e artigos afirmam que as estratégias organizacionais são importantes. O autor apresenta uma revisão das diferentes perspectivas de investigação estratégicas, sugerindo que a perspectiva adaptativa é a que proporciona uma melhor contribuição para seu entendimento, porém recomenda que seja mais prudente utilizar uma integração das características das diferentes perspectivas, a fim de superar as limitações de um único modelo.

Dessa maneira, após destacar a importância de estudar o comportamento estratégico das organizações, este artigo verifica quais os comportamentos adotados pelos administradores de hotéis em Florianópolis, SC, segundo a tipologia de Miles e Snow.

\section{COMPORTAMENTO ESTRATÉGICO}

Muitas teorias estudaram o comportamento organizacional, segundo Miles e Snow (1978), no entanto nenhuma delas, conforme os autores, contempla todas as formas de comportamento encontradas nas organizações. Este fato ocorre porque as organizações são muito diversificadas, mutáveis e complexas.

Caves e Porter (1977), ao abordarem os aspectos das mudanças de comportamento organizacional, sugerem que as decisões que implicam comportamentos estratégicos a serem adotados pelas organizações são ainda mais importantes quando estão em um mercado altamente competitivo, fragmentado e com profundas mudanças.

Grant (1996) propõe que, como a estratégia está relacionada tanto ao crescente dinamismo quanto à complexidade organizacional, o comportamento seria a base desse processo, possibilitando que os administradores definam seus objetivos com base em suas experiências anteriores, muitas vezes pressionadas por situações extremamente complexas.

Ghemawat (2000) sugere que pesquisas sobre o comportamento estratégico dos administradores, que levam às mudanças organizacionais de sucesso, indicam forte senso de insatisfação com o status quo, um poderoso indicativo daquilo que pode ser realizado, que, muitas vezes, envolve mudanças de pessoas e da estrutura organizacional.

Apesar de muito ter-se falado que as empresas devem pensar e agir taticamente, existem, ainda, muitas falhas da alta administração em atuar de maneira estratégica. Este fato é comprovado por Rossetto (1998), em trabalho realizado no setor da construção civil. O autor ressalta que, no processo de formulação das estratégias, o líder não agiu de forma articulada, mas sim de maneira não planejada, sendo que as estratégias surgiram, na sua grande maioria, de mudanças emergentes. Para gerenciar esse processo, ele não planejou estratégias, mas reconheceu e interveio quando necessário.

A pesquisa de Costa e Teare (2000) comparando a atuação de redes hoteleiras portuguesas constatou que os comportamentos investigativos eram mais frequentes naquelas organizações onde havia estratégias formalizadas do que naquelas em que a estratégia era desenvolvida informalmente, através apenas da visão dos altos executivos.

Em trabalho realizado na Índia, durante a liberação econômica do país, Ray Sougata (2004) estudou o comportamento estratégico adotado pelas empresas e verificou que aquelas 
que reconheceram antecipadamente as mudanças, favoráveis ou não, aumentaram sua escala de negócios, promoveram a diversificação, reorganizaram sua carteira de clientes e modernizaram suas instalações e tecnologia, alcançando desempenho superior em relação às demais.

Para Olson, Slater e Hult (2005), o desempenho da firma é determinado pela forma eficaz e eficiente de implementar suas estratégias de negócios, sobretudo a adoção de comportamentos de orientação ao cliente, a análise de seus concorrentes, a percepção e adoção das inovações pertinentes e a forma de lidar com os custos de gestão. Os autores estudaram o comportamento de 222 gestores e verificaram que, para cada tipo de estratégia a ser adotada, existem diferentes tipos de comportamentos de sucesso.

Zúñiga-Vicente e Vicente-Lorente (2006) estudaram o efeito de movimentos estratégicos ou mudança estratégica e a probabilidade de sobrevivência de bancos espanhóis que se submeteram a transformações radicais em seus ambientes. Os autores testaram duas perspectivas teóricas: a visão de adaptação (gerenciamento estratégico clássico e capacidades dinâmicas) e a abordagem ecológica. Os resultados confirmaram a sobreposição das unidades que atuavam com os modelos estratégicos clássicos, em relação aos que se utilizavam da abordagem ecológica, como aqueles com maior probabilidade de sobrevivência organizacional.

Nesse sentido, percebe-se que o propósito da estratégia é especificar os tipos de comportamentos a serem adotados para sua adaptação ou mudança. Portanto, o entendimento do comportamento, objeto de estudo deste trabalho, passa pelo entendimento da estratégia organizacional, uma vez que esta guiará as decisões necessárias para o alcance da visão de futuro.

O trabalho de Miles e Snow (1978), muito utilizado para a compreensão da estratégia organizacional, classifica as posturas estratégicas em prospectoras, analistas, defensivas e reativas. Ao apoiar-se nesses autores, Hambrick (1983) examinou as tendências do desempenho dos estilos prospector e defensor em ambientes diferentes e concluiu que tanto o prospector quanto o defensor diferiam em suas tendências de desempenho dependendo da natureza do ambiente, ou seja, a escolha da estratégia deve ser em função das exigências do ambiente e do tipo de desempenho que se deseja naquele momento.

Outros autores que se basearam em Miles e Snow (1978) foram Conant, Mokwa e Varadarajan (1990). Para eles, embora haja muitos trabalhos que tenham se utilizado do conhecimento sobre os arquétipos estratégicos, há, ainda, a necessidade de mais pesquisas na área, sobretudo porque muitas das pesquisas realizadas procuram excluir de sua análise o tipo reativo.

Para Zahra e Pearce II (1990), a tipologia de Miles e Snow é uma das mais populares classificações de estratégias ao nível de negócios. Consequentemente, grande atenção é dada ao exame da validade de pesquisas usadas na sua defesa. Zahra e Pearce II (1990) realizaram uma análise de pesquisas sobre o uso da tipologia com foco em quatro questões: na identificação da natureza dos tipos estratégicos; testando as previsões tipológicas no que concerne a dimensões do "ciclo adaptativo"; na ligação entre estratégia e ambiente; e nas diferenças de desempenho entre tipos estratégicos.

Gimenez et al. (1999) verificaram, em 107 empresas de Londrina, PR, as escolhas estratégicas adotadas pelos gestores, constatando que havia ocorrência dos quatro tipos estratégicos de Miles e Snow, sendo o desempenho do tipo reativo o menos eficaz em termos de crescimento organizacional, e que havia, nessas empresas, uma frequência maior do tipo analista.

Gimenez (2000) salienta que, apesar de a tipologia de Miles e Snow ter sido desenvolvida há algum tempo (1978), ela tem grande importância para a compreensão do comportamento organizacional, porque especifica o relacionamento entre estratégia, estrutura e processos de uma forma que permite a identificação das organizações como um todo em interação com seus ambientes. Segundo o autor, a teoria de Miles e Snow tem sido amplamente testada nos mais diversos ambientes empresariais e tem denotado possuir qualidades muito boas em termos de codificação e predição. 
Slater e Olson (2001) associaram a tipologia de Miles e Snow às estratégias de marketing em unidades estratégicas de negócios. A taxonomia desenvolvida pelos autores foi denominada marqueteiros agressivos, marqueteiros de massa, minimizadores de marketing e marqueteiros de valor. As performances superiores foram alcançadas por aqueles que associaram fortemente as estratégias de marketing com os tipos estratégicos de Miles e Snow.

Hambrick (2003) confirma a importância da tipologia de Miles e Snow pelo profundo efeito causado nos campos do gerenciamento estratégico e da teoria organizacional. Segundo o autor, estudiosos têm confiado profundamente nessa tipologia para o desenvolvimento de ferramentas práticas, e muitos gerentes têm sido influenciados por ela.

Castle (2003) utilizou a tipologia de Miles e Snow para averiguar as respostas estratégicas de enfermeiros atuando em ambiente de mudança, verificando se as respostas estratégicas estavam associadas às performances e à qualidade dos serviços prestados pelos enfermeiros. $O$ autor percebeu que aqueles que adotaram a postura prospectora tiveram maiores resultados de qualidade, seguidos pelos defensores, analistas e reativos.

Trabalhos mais recentes, utilizando-se da tipologia de Miles e Snow, foram realizados por Arragón-Sanchez e Sánchez-Marín (2005) em empresas espanholas. Os autores investigaram a orientação estratégica e suas consequências para a eficiência empresarial, relacionando esta orientação com aspectos gerenciais, como: posição tecnológica, inovação, estrutura orgânica e gestão de recursos humanos.

A tipologia de Miles e Snow também serviu de modelo para que alguns autores desenvolvessem suas próprias tipologias. Desarbo et al. (2005) criaram seu próprio modelo de pesquisa, também formado por quatro tipos estratégicos, que foram assim definidos: grupo 1, formado por firmas prospectoras com forças tecnológicas, mas com relativas fraquezas mercadológicas, exemplificado por um modelo empresarial asiático; grupo 2, formado por firmas defensivas com habilidades de marketing, que operam em ambientes relativamente incertos; grupo 3, formado por firmas que unem forças mercadológicas e gerenciais, exemplificado pelo modelo empresarial americano, em que se encontram firmas que apresentam mais características analíticas, mas com algumas características prospectoras e defensoras; e grupo 4, formado uniformemente por empresas prospectoras e analíticas, exemplificado por empresas que apresentam características tanto americanas como asiáticas, pois estas não são fortes somente em marketing e gestão, mas também em capacidades tecnológicas. Este último grupo foi chamado pelos autores de firmas equilibradamente prospectoras.

Outro autor que se utilizou do modelo de Miles e Snow foi Gurkov (2007). Ele combinou a tipologia de Miles e Snow com a abordagem de Bowman e Faulkner (1997), desenvolvendo posturas que envolveram aspectos da competitividade e inovação e que denominou de:

- outsider: baixa qualidade, preço, inovação, poucas competências e alto custo;

- coast defender: baixa qualidade, preço, custo, poucas competências e moderada inovação;

- quality defender: alta qualidade, preço e competências e custos e inovação moderada;

- analyzer: alta qualidade, preço, competências e custos e moderada inovação;

- prospector: alta qualidade, competências e inovação e preço e custo baixos; e

- monopolist: qualidade e preço relativos, custo e competências variáveis e inovação reprimida. Esse modelo foi aplicado, através de uma survey, em cerca de 600 firmas russas, para descrever os arquétipos estratégicos dominantes no país.

Ao referenciar o uso de uma determinada tipologia na realização de uma pesquisa, Hambrick (1984) salienta que ela busca concentrar o foco e melhor relacionar as variáveis 
encontradas e que a importância da utilização de tipologias está na redução do vasto leque de combinações que um pesquisador teria que considerar.

A tipologia de comportamento estratégico de Miles e Snow, utilizada neste trabalho, pode ser mais bem compreendida a partir de cinco características básicas: a coalizão dominante, a percepção dos membros da coalizão dominante, a segmentação, o monitoramento do ambiente externo e as restrições dinâmicas.

A coalizão dominante é constituída pelas pessoas responsáveis pela tomada de decisão organizacional e tem como responsabilidade a busca da solução dos problemas organizacionais. A percepção do ambiente organizacional, por sua vez, é dada pela interpretação que a coalizão dominante tem deste mesmo ambiente. Já a segmentação corresponde à fragmentação ou à partição do ambiente a partir da percepção dos decisores organizacionais e da distribuição dos componentes ambientais às várias unidades organizacionais, os quais serão alocados de acordo com a sua importância estratégica. O monitoramento do ambiente externo refere-se à responsabilidade da coalizão dominante em vigiar aqueles elementos do ambiente que são mais críticos para a organização. O exame desses elementos é que constituirá a base para as opções estratégicas da organização.

As restrições dinâmicas, por fim, segundo Miles e Snow (1978), são aqueles fatores relativos ao passado da organização e aos aspectos organizacionais atuais, como a estratégia, a estrutura e o desempenho, que acabam limitando as ações organizacionais. As restrições existentes podem ser atenuadas ou removidas por meio da alteração da estratégia, sendo que qualquer outra direção escolhida trará em seu bojo novas restrições.

Existe outro fator, para Miles e Snow (1978), que também contribui para a melhor compreensão do processo de escolha estratégica ou de adaptação organizacional, a análise do ciclo adaptativo. Os autores explicam que esse ciclo, embora existente em todas as organizações, seja, talvez, melhor identificado em novas organizações ou naquelas que passam por rápido crescimento, ou ainda nas que saíram recentemente de uma grande crise.

O dinâmico processo de adaptação organizacional (ciclo adaptativo), de acordo com Miles e Snow (1978), pode ser separado em três problemas que os administradores devem constantemente resolver:

a) problema empresarial: tanto em novas quanto em já existentes organizações, a solução para o problema empreendedor é indicada pela aceitação gerencial de um particular domínio de produto-mercado. Esta aceitação se torna evidente quando o administrador decide comprometer recursos para atingir objetivos relativos à solução empresarial;

b) problema de engenharia: envolve a criação de um sistema que ponha em operação a solução administrativa para o problema empreendedor. A criação de cada sistema requer gerenciamento para selecionar uma tecnologia apropriada para produção e distribuição dos produtos/serviços escolhidos;

c) problema administrativo: envolve a redução da incerteza no sistema organizacional ou a racionalização e estabilização das atividades que prosperamente resolveram problemas enfrentados pela organização durante as fases empreendedora e de engenharia. Também envolve a formulação e implementação de processos que permitirão à organização continuar evoluindo.

Os autores enfatizam o que seria uma filosofia de comportamento da organização, baseada no tratamento de problemas empreendedores, de engenharia e administrativos. Cada um desses problemas exige uma configuração particular de tecnologia, estrutura e processos, consistente com sua estratégia de mercado. Essa particularidade pode ser mais bem visualizada na figura 1. 


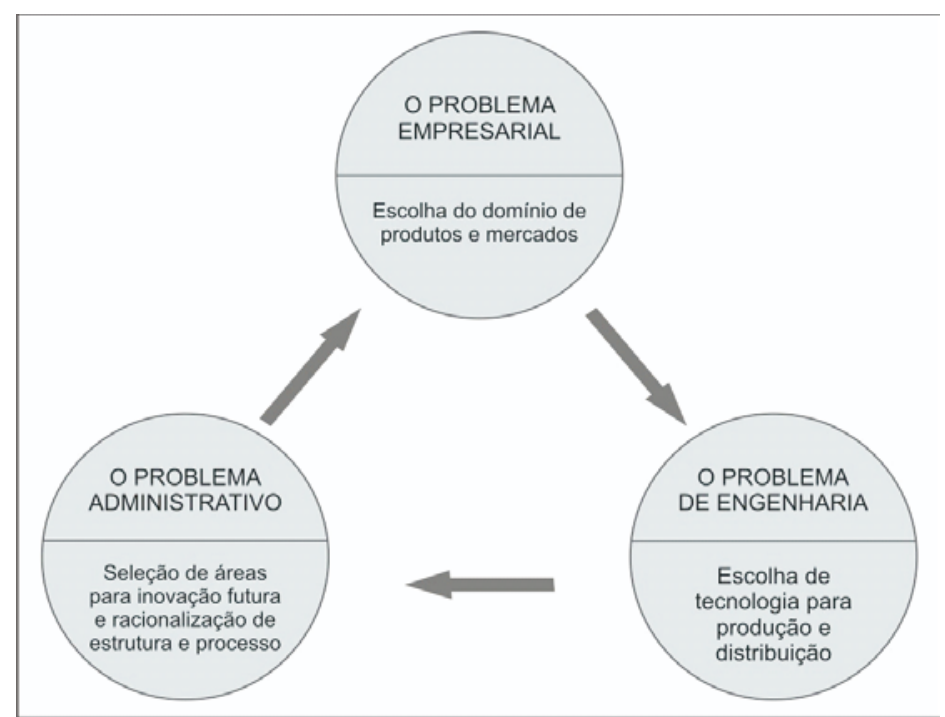

Figura 1 - Ciclo adaptativo

Fonte: Miles e Snow (1978, p. 24).

Com o propósito de adaptação da organização ao seu ambiente, o modelo estratégico desenvolvido por Miles e Snow (1978) focaliza o processo de adaptação organizacional, expresso através da inter-relação entre estratégia, estrutura e processos. Dois elementos básicos compõem esse modelo: uma abordagem geral do processo de adaptação, especificando as principais linhas de decisão que uma empresa deve tomar, de forma a se manter alinhada e ajustada ao seu ambiente; e uma tipologia organizacional, retratando os diferentes padrões de comportamento adaptativo utilizados pelas empresas.

Essa tipologia classifica as unidades de negócio em quatro categorias: prospectoras, defensoras, analisadoras e reativas, conforme quadro 1.

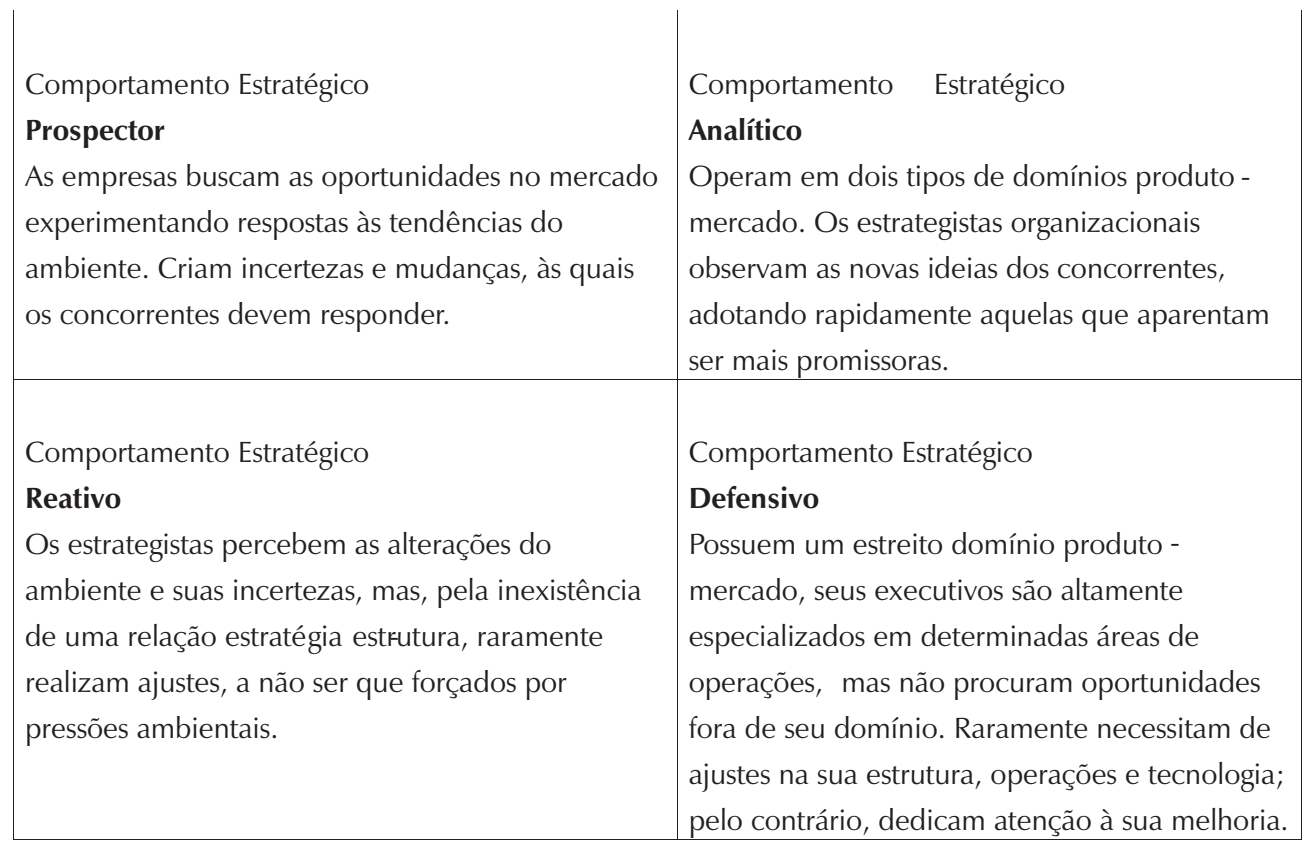

Quadro1- Tipologia de Miles e Snow (1978)

Fonte: Miles e Snow (1978). 
Os prospectores tendem a se basear em uma estrutura organizacional com controle mais descentralizado e orientado para produtos, favorecendo o desenvolvimento de tecnologias. A necessidade de identificar novas oportunidades os obriga a desenvolver capacidades e mecanismos para observar o ambiente, os acontecimentos e as tendências. Uma vez que as atividades de varredura não se limitam ao seu domínio corrente, os prospectores tendem a ser os precursores de inovações. A reputação de inovador é normalmente essencial para o sucesso do prospector, à medida que lhe permite cativar os clientes ávidos por novidades (MILES; SNOW, 1978).

Para o prospector, o problema empreendedor de como localizar e explorar novas oportunidades de produto e mercado é preponderante, ficando a solução condicionada ao composto de produtos atual e futuro. O problema de engenharia reside em como evitar comprometimentos de longo prazo com relação a uma dada tecnologia, o que pode ser resolvido com a utilização de múltiplas tecnologias, com baixo emprego de rotina e mecanização. Já o problema administrativo está em facilitar as operações, em vez de controlá-las. Assim, o sistema administrativo deve ser capaz de distribuir e coordenar recursos entre as unidades de negócio e os projetos e não de planejar e controlar as operações da empresa como um todo.

Os defensores apresentam uma visão mais conservadora no tocante ao desenvolvimento de produtos e mercados e procuram garantir uma posição segura e estável no seu domínio produto-mercado. Raramente introduzem novidades tecnológicas, procurando competir em dimensões como preço ou qualidade dos produtos atuais, delimitando e isolando para si um espaço estável do mercado.

Seu problema de engenharia está na produção de bens e serviços da forma mais eficiente possível e pode ser resolvido através da adoção de uma tecnologia núcleo, altamente eficiente. O problema administrativo de como alcançar controle severo da organização, de forma a garantir eficiência, é tratado através da implementação de controles centralizados e da ênfase em determinadas áreas funcionais, como as de finanças e produção. O grande risco do defensor reside na possibilidade de o ambiente sofrer uma mudança para a qual sua tecnologia não esteja adaptada.

Já os analisadores são uma mistura de prospectores e defensores: ao mesmo tempo em que se envolvem na exploração de novos produtos e mercados, também procuram manter um domínio estável de produtos e clientes, a partir de suas principais competências e habilidades, além de procurarem minimizar o risco sem abrir mão de oportunidades. Seu problema empresarial está em como identificar e explorar novas oportunidades de produto e mercado, mantendo uma base firme de produtos e clientes tradicionais.

O problema de engenharia passa a ser o de como alcançar e garantir um equilíbrio entre as demandas conflitantes por flexibilidade e estabilidade tecnológica. Similarmente, seu problema administrativo é o de como criar estruturas e processos diferenciados, de forma a acomodar, ao mesmo tempo, as áreas dinâmicas e estáticas da empresa. Os principais riscos incorridos pelo analisador referem-se à possível ineficiência advinda da adoção de tecnologias diferenciadas.

Por fim, os reativos representam um "fracasso" estratégico, à medida que exibem inconsistências entre sua estratégia e sua tecnologia, estrutura e processos. Miles e Snow (1978) acreditam que eles apresentam um desempenho inferior ao dos demais tipos estratégicos puros.

Esse tipo estratégico somente se sustentaria, no tempo, em ambientes monopolíticos ou regulados. Em outros ambientes ou a empresa implementa adequadamente um dos outros tipos estratégicos ou sucumbe às pressões dos concorrentes e dos clientes. Não é um tipo estratégico 
puro, mas residual, não congruente com a postura de resposta ao ambiente. A ausência de uma orientação consistente faz com que eles reajam às pressões ambientais apenas quando forçados.

Para Miles e Snow (1978), a sua tipologia pode compreender o comportamento estratégico de cada organização, com a predominância de um tipo específico. Salienta-se, porém, que as empresas não são puramente um dos tipos determinados pelos autores. O comportamento puro existe apenas para bases conceituais, mas, na prática, o comportamento é bem mais complexo, fazendo com que a classificação seja realizada em termos de predominância de padrões de comportamento.

\section{METODOLOGIA}

Esta pesquisa se caracteriza por um estudo quantitativo do tipo survey e, quanto aos fins, foi descritiva. A população consultada foi de 22 hotéis situados em Florianópolis, classificados entre três e cinco estrelas.

Os dados foram coletados por meio de questionário com perguntas fechadas, o que permitiu o acesso às informações necessárias para a consecução deste trabalho, como a identificação do comportamento estratégico, classificado como prospector, analista, reativos e defensivo. Um questionário deve, segundo Malhotra (2001), possibilitar o entendimento das questões, devendo, para isso, ter forma e vocabulário adequados aos propósitos da mensuração, e a medida deve representar a substância ou o conteúdo daquilo que se quer medir.

O questionário aplicado, que identificou o comportamento estratégico adotado pelos administradores de hotéis, em um período de cinco anos, teve como base o modelo adaptado de Conant, Mokwa e Varadarajan (1990), dos tipos estratégicos de Miles e Snow.

Ao referenciar o uso de uma determinada tipologia na realização de uma pesquisa, Hambrick (1984) salienta que ela busca concentrar o foco e melhor relacionar as variáveis encontradas e que a importância da utilização de tipologias está na redução do vasto leque de combinações que um pesquisador teria que considerar. Segundo o autor, as categorias de uma tipologia representam caracteres gerais que definem um pacote de atributos, ou seja, uma vez identificada, cada variável pode ser testada e expandida por pesquisadores que tendem a revelar sua preferência por um determinado conjunto dessas variáveis.

Para a organização dos dados, foi utilizado o software Excel e o Statistica 6.0. A planilha eletrônica Excel possibilitou a elaboração de tabelas e gráficos e, pela facilidade de operacionalização, permitiu a importação da tabela de dados para o Statistica, com o qual se efetuou a análise em componentes principais, enquanto método da análise multivariada. A análise multivariada é um conjunto de métodos estatísticos que permite a análise simultânea de medidas múltiplas para cada indivíduo ou objeto em análise, sendo que o método de componentes principais possibilitou reconhecer as associações entre as variáveis que se desejava estudar e as semelhanças entre os hotéis.

Já o método da análise dos componentes principais, segundo Reis (1997), é um procedimento estatístico multivariado que permite transformar um conjunto de variáveis iniciais, correlacionadas entre si, em um outro conjunto de variáveis hipotéticas não correlacionadas (ortogonais). A análise se efetua por meio da criação de novas variáveis hipotéticas, resultantes de combinações lineares das variáveis originais que, por restrições no procedimento algébrico, são ordenadas de maneira decrescente, segundo a informação que recuperam da base de dados, e são geradas para serem independentes entre si. Ainda, no método de componentes 
principais, podem-se distinguir, na análise fatorial, os fatores comuns e específicos, sendo seu objetivo principal explicar, da melhor forma possível, a variância original no conjunto de dados por alguns componentes principais (HAIR JR et al., 2005).

Dessa forma o modelo fatorial dos componentes principais possibilitou, neste trabalho, que a matriz de dados fosse decomposta em duas outras matrizes, uma relativa às variáveis e chamada matriz de cargas fatoriais e outra relacionada aos casos ou hotéis, chamada matriz de escores fatoriais, permitindo reconhecer as associações entre as variáveis que se desejava estudar e as semelhanças entre os hotéis.

\section{ANÁLISE DO COMPORTAMENTO}

Nesta seção, será realizada a análise dos comportamentos estratégicos adotados pelos administradores de hotéis. Para que se tenha um melhor detalhamento das informações, serão, primeiramente, analisados separadamente os comportamentos adotados segundo o ciclo adaptativo de Miles e Snow, que estão relacionados aos três problemas aos quais se referem: empresarial, de engenharia e administrativo. Posteriormente, será apresentado o comportamento geral adotado pelos administradores.

A primeira dimensão a ser analisada é a dimensão empresarial, que está relacionada aos aspectos do domínio de produto-mercado, postura de sucesso, monitoramento ambiental e crescimento, ou seja, como os hotéis pesquisados se posicionam frente ao mercado em que atuam.

Os dados para o problema empresarial estão representados na tabela 1 e no gráfico 1, representativos do comportamento adotado pelos administradores de hotéis.

Tabela 1 - Problema empresarial

\begin{tabular}{|c|c|c|c|c|c|c|c|c|}
\hline \multirow{2}{*}{$\begin{array}{l}\text { Problema } \\
\text { empresarial }\end{array}$} & \multicolumn{2}{|c|}{ Prospector } & \multicolumn{2}{|c|}{ Analista } & \multicolumn{2}{|c|}{ Reativo } & \multicolumn{2}{|c|}{ Defensivo } \\
\hline & Freq. & $\%$ & Freq. & $\%$ & Freq. & $\%$ & Freq. & $\%$ \\
\hline $\begin{array}{l}\text { Domínio de } \\
\text { produto- } \\
\text { mercado }\end{array}$ & 8 & $36 \%$ & 2 & $9 \%$ & 7 & $32 \%$ & 5 & $23 \%$ \\
\hline $\begin{array}{l}\text { Postura de } \\
\text { sucesso }\end{array}$ & 4 & $18 \%$ & 12 & $54,5 \%$ & 5 & $23 \%$ & 1 & $4,5 \%$ \\
\hline $\begin{array}{l}\text { Monitoramento } \\
\text { ambiental }\end{array}$ & 8 & $36.5 \%$ & 8 & $36.5 \%$ & 3 & $13,5 \%$ & 3 & $13.5 \%$ \\
\hline Crescimento & 10 & $45,5 \%$ & 10 & $45,5 \%$ & 1 & $4,5 \%$ & 1 & $4,5 \%$ \\
\hline GERAL & & $34 \%$ & & $37 \%$ & & $18 \%$ & & $11 \%$ \\
\hline
\end{tabular}

Fonte: dados da pesquisa. 


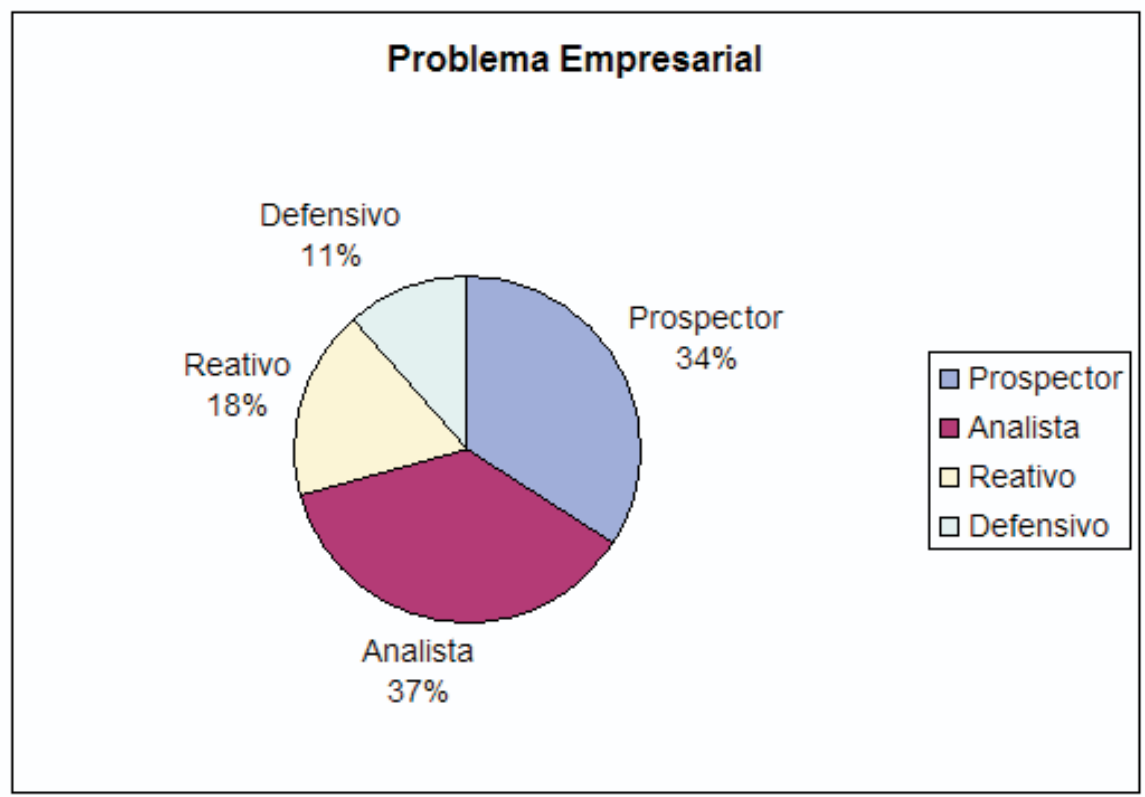

Gráfico 1 - Problema empresarial

Fonte: dados da pesquisa.

Resultado 1: a análise conjunta das dimensões do problema empresarial demonstrou que houve uma predominância do comportamento estratégico analista, sendo seguido pelos comportamentos prospector, reativo e defensivo.

A segunda dimensão apresentada é aquela que tem relação com o problema de engenharia, sendo que esse problema envolve a criação de um sistema que ponha em operação a solução administrativa para o problema empreendedor, já que cada sistema requer gerenciamento para selecionar uma tecnologia apropriada para produção e distribuição dos produtos/serviços escolhidos. Essa dimensão abrange os objetivos tecnológicos, a amplitude tecnológica e o anteparo tecnológico e está representada pela tabela 2 e gráfico 2.

Tabela 2 - Problema de engenharia

\begin{tabular}{|c|c|c|c|c|c|c|c|c|}
\hline \multirow{2}{*}{$\begin{array}{l}\text { Problema de } \\
\text { engenharia }\end{array}$} & \multicolumn{2}{|c|}{ Prospector } & \multicolumn{2}{|c|}{ Analista } & \multicolumn{2}{|c|}{ Reativo } & \multicolumn{2}{|c|}{ Defensivo } \\
\hline & Freq. & $\%$ & Freq. & $\%$ & Freq. & $\%$ & Freq. & $\%$ \\
\hline $\begin{array}{l}\text { Objetivos } \\
\text { tecnológicos }\end{array}$ & 4 & $18 \%$ & 15 & $68,5 \%$ & 3 & $13,5 \%$ & 0 & 0 \\
\hline $\begin{array}{l}\text { Amplitude } \\
\text { tecnológica }\end{array}$ & 12 & $54,5 \%$ & 1 & $4,5 \%$ & 6 & $27,5 \%$ & 3 & $13,5 \%$ \\
\hline $\begin{array}{l}\text { Anteparo } \\
\text { tecnológico }\end{array}$ & 9 & $41 \%$ & 4 & $18,5 \%$ & 3 & $13,5 \%$ & 6 & $27 \%$ \\
\hline GERAL & & $38 \%$ & & $30 \%$ & & $18 \%$ & & $14 \%$ \\
\hline
\end{tabular}




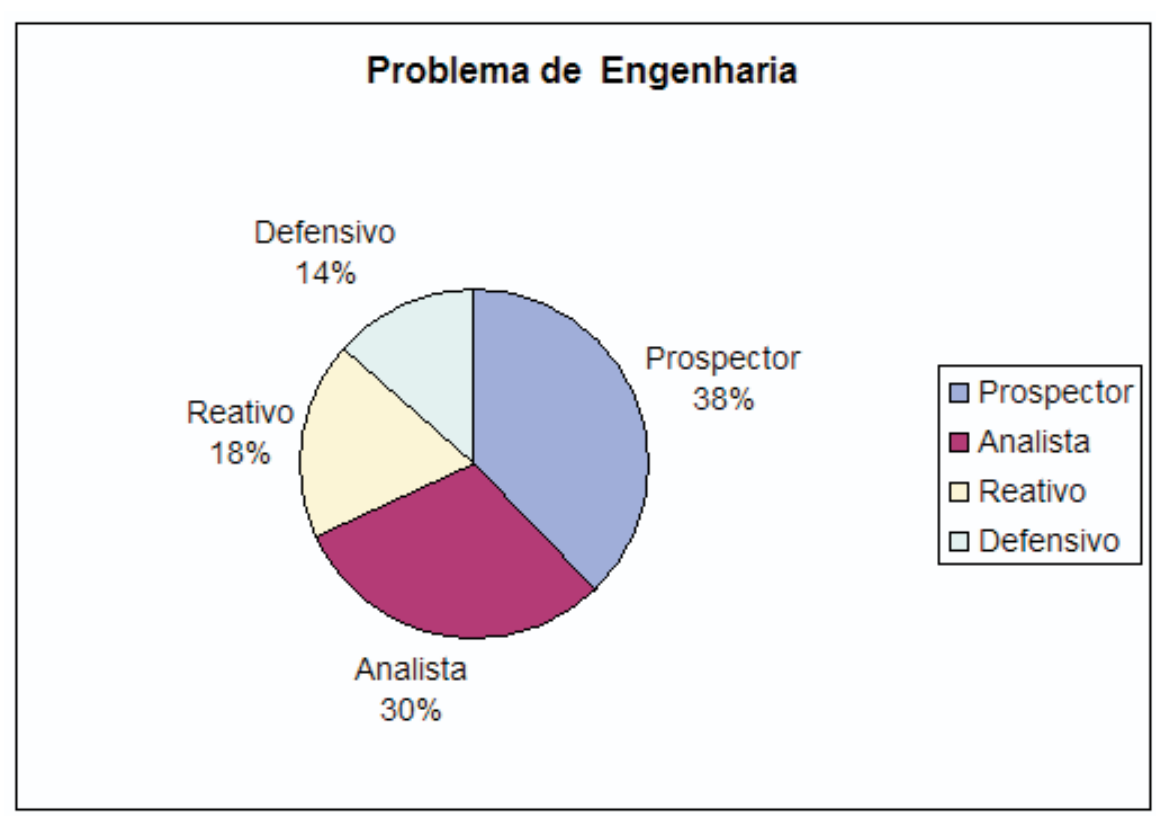

Gráfico 2 - Problema de engenharia

Fonte: dados da pesquisa.

Resultado 2: em análise conjunta das dimensões do problema de engenharia, percebeu-se que houve uma predominância do comportamento estratégico prospector, seguido pelos comportamentos analista, reativo e defensivo.

Por último, são analisados os problemas administrativos, que consistem em reduzir a incerteza no sistema organizacional ou racionalizar e estabilizar atividades que prosperamente resolveram problemas enfrentados pela organização durante as fases empreendedoras e de engenharia, o que também envolve formular e implementar processos que permitirão à organização continuar evoluindo. Esse problema é composto pelas dimensões coalizão dominante, planejamento, estrutura e controle e está representado pela tabela e gráfico 3.

Tabela 3 - Problema administrativo

\begin{tabular}{l|c|c|c|c|c|c|c|c|}
\hline \multirow{2}{*}{$\begin{array}{l}\text { Problema } \\
\text { administrativo }\end{array}$} & \multicolumn{2}{|c|}{ Prospector } & \multicolumn{2}{c|}{ Analista } & \multicolumn{2}{c|}{ Reativo } & \multicolumn{2}{c|}{ Defensivo } \\
\cline { 2 - 9 } & Freq. & $\%$ & Freq. & $\%$ & Freq. & $\%$ & Freq. & $\%$ \\
\hline $\begin{array}{l}\text { Coalizão } \\
\text { dominante }\end{array}$ & 8 & $36 \%$ & 8 & $36 \%$ & 1 & $4,5 \%$ & 5 & $23,5 \%$ \\
\hline Planejamento & 10 & $45,5 \%$ & 3 & $13,5 \%$ & 2 & $9 \%$ & 7 & $32 \%$ \\
\hline Estrutura & 8 & $36,5 \%$ & 8 & $36,5 \%$ & 3 & $13,5 \%$ & 3 & $13,5 \%$ \\
\hline Controle & 9 & $41,5 \%$ & 5 & $22,5 \%$ & 2 & $9 \%$ & 6 & $27 \%$ \\
GERAL & & $40 \%$ & & $27 \%$ & & $9 \%$ & & $24 \%$ \\
\hline
\end{tabular}

Fonte: dados da pesquisa. 


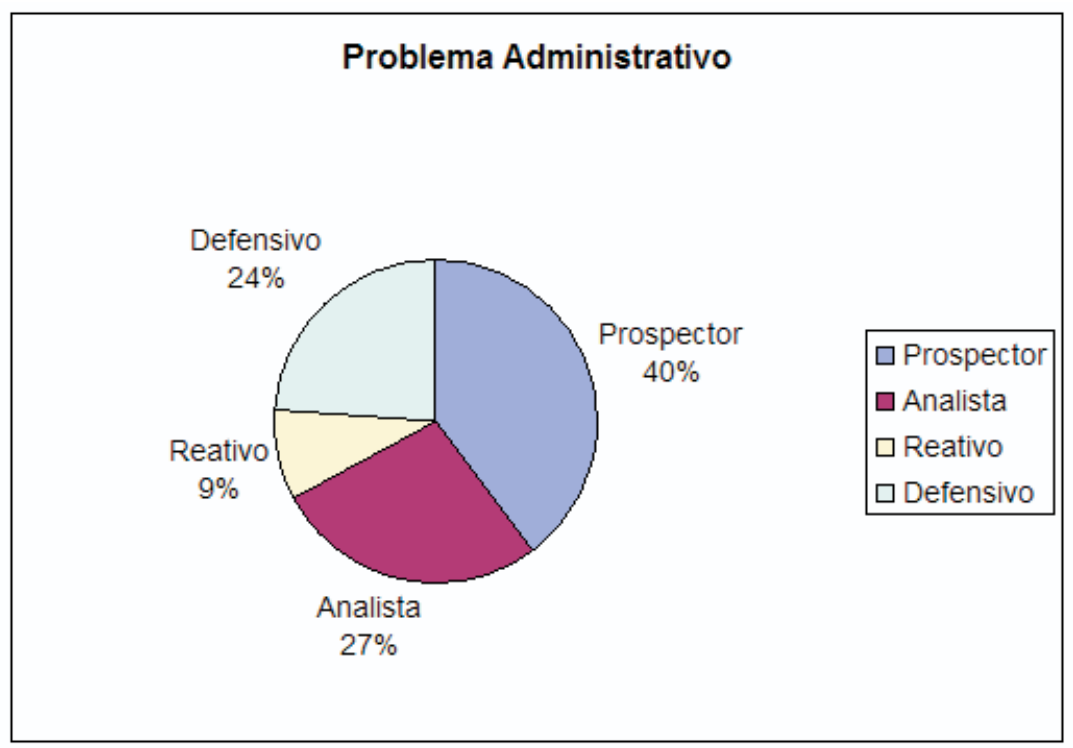

Gráfico 3 - Problema administrativo

Fonte: dados da pesquisa.

Resultado 3: a análise conjunta das dimensões do problema administrativo demonstrou que houve uma predominância do comportamento estratégico prospector, seguido pelos comportamentos analista, reativo e defensivo.

A partir do levantamento dos dados dos problemas do ciclo adaptativo de Miles e Snow, pode-se realizar uma síntese dos problemas empresarial, de engenharia e administrativo, percebendo-se que houve uma sobreposição do comportamento prospector sobre os demais. Isto implica dizer que são predominantes as atitudes criativas e inovadoras para entrar em novos mercados, que existe a disponibilidade dos recursos necessários para a criação de novos produtos e serviços e para entrar em novos mercados, bem como existe a preocupação com e a capacidade para reduzir as incertezas, racionalizar e estabilizar as atividades que possam resolver os problemas enfrentados nas fases empresarial e de engenharia.

Entretanto, também houve uma grande incidência de repostas que identificam o comportamento analista, sendo que teve destaque para as variáveis que estão relacionadas às questões de objetivos tecnológicos. Este fato faz com que os administradores deem prioridade à manutenção dos custos sob controle e, seletivamente, à geração de novos produtos e serviços e à entrada em novos mercados.

Quanto aos demais comportamentos, em uma análise geral, pode-se notar que não houve, em nenhum momento, tanto para o reativo quanto para o defensivo, uma sobreposição sobre os comportamentos prospector ou analista. Porém, quanto ao comportamento reativo, pode-se dizer foi dada uma atenção maior para os aspectos relacionados com os serviços que estavam sendo modificados conforme as necessidades do mercado e de desenvolvimento de habilidades em relação aos termos que estejam próximos dos clientes e mercados. A mesma análise pode ser feita em relação ao comportamento defensivo, porém para as variáveis que envolvem as questões relacionadas com as habilidades em realizar um limitado número de atividades bem feitas, identificar soluções para os atuais problemas e se manter no mercado e ter as decisões altamente centralizadas através de relatórios de faturamento. 
Em relação ao comportamento geral, adotado pelos administradores de hotéis de Florianópolis nos últimos cinco anos, ele pode ser visualizado na tabela 4 e no gráfico 4.

Tabela 4 - Comportamentos adaptativos

\begin{tabular}{lccc|c}
\hline Comportamento & Freq. & $\begin{array}{l}\text { Freq. } \\
\text { acumulada }\end{array}$ & Freq. relativa & Freq. relativa acumulada \\
\hline Prospector & 10 & 10 & $45,5 \%$ & 45,5 \\
Analista & 7 & 17 & $31,5 \%$ & 77 \\
\hline Reativo & 4 & 21 & $18,5 \%$ & 100 \\
\hline Defensivo & 1 & 22 & $4,5 \%$ & \\
\hline \multicolumn{2}{c}{ Fonte: dados da pesquisa. }
\end{tabular}

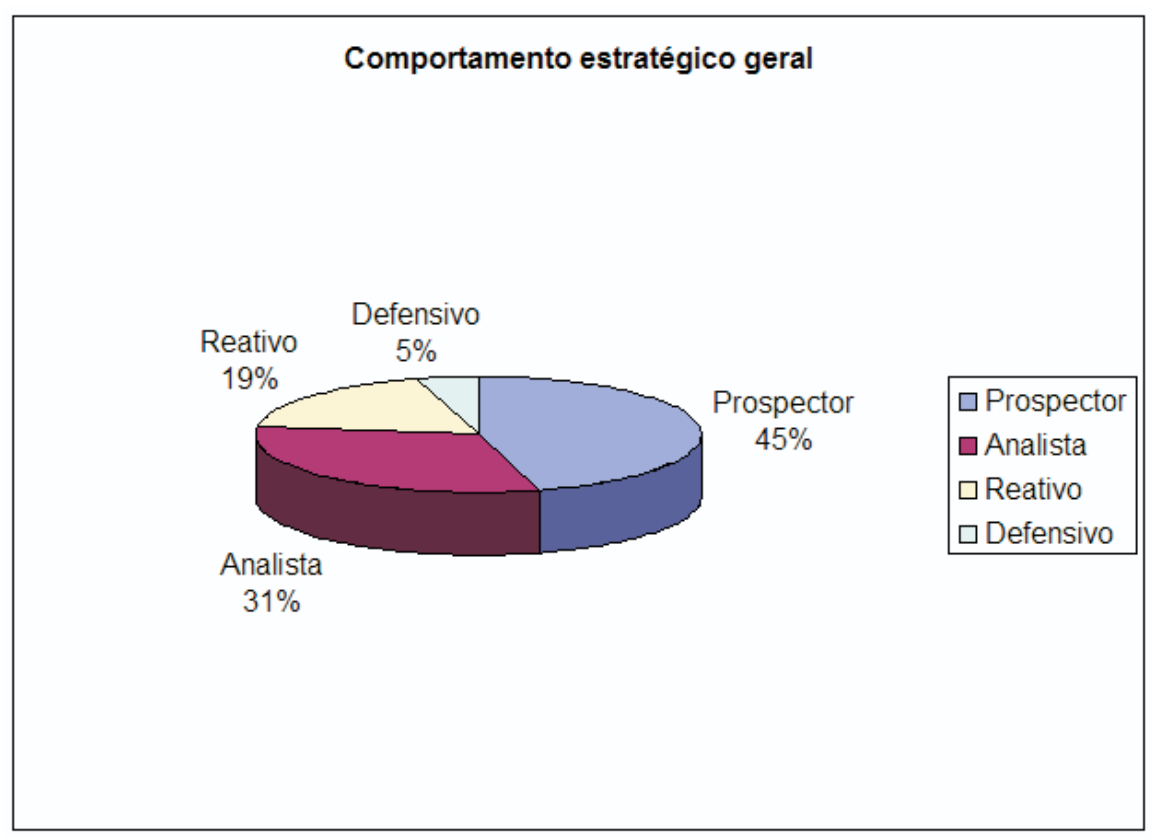

Gráfico 4 - Comportamento estratégico geral Fonte: dados da pesquisa.

Percebe-se que, de um modo geral, para os comportamentos adotados pelos administradores de hotéis de Florianópolis, existe uma preponderância do comportamento prospector, com uma frequência de 45,5\%, sendo seguido pelo comportamento analista, com 31,5\%, reativo, com $18,5 \%$ e pelo comportamento defensivo, com apenas $4,5 \%$.

Esses dados permitem supor que, em grande parte, os administradores de hotéis estão atentos para as mudanças ambientais e adotam uma postura agressiva em relação ao mercado, buscando oportunidades, investindo na criação de novos produtos e serviços e entrando em novos mercados. Seguindo esse comportamento majoritário, segue-se uma postura de precaução, mantendo os mercados existentes e somente entrando em novos mercados com novos 
produtos e serviços após estes serem bem analisados. Outra parcela dos administradores procura adotar uma postura de reação frente às ações dos concorrentes, não adotando nenhuma postura proativa por falta de estratégias e, em função disso, tornando-se vulneráveis aos ataques impostos pela concorrência. Por fim, uma pequena parcela dos comportamentos dos administradores demonstra que eles dão prioridade à defesa do mercado já conquistado e à posição alcançada e, em vez de investir em novas tecnologias, procuram manter uma posição que lhes permita competir através de preços e qualidade.

Essa análise é congruente com a análise do ciclo adaptativo em que foram detalhadas as posturas adotadas em relação aos problemas empresarial, de engenharia e administrativo, realizada anteriormente, na qual se obteve, também, uma incidência maior do comportamento prospector.

Resultado 4: os administradores de hotéis em Florianópolis adotam a postura prospectora como comportamento estratégico geral.

\section{CONCLUSÃO}

Os resultados encontrados nesta pesquisa, em relação ao comportamento estratégico adotado pelos administradores de hotéis de Florianópolis, apontaram a predominância do comportamento prospector, com uma frequência de $45,5 \%$, sendo seguido pelo comportamento analista, com $31,5 \%$, reativo, com $18,5 \%$, e defensivo, com somente $4,5 \%$.

A entrada das grandes redes que, por terem experiências de outros mercados e estarem atentas às oportunidades que surgem com a crescente entrada de turistas na cidade, somados à mudança do perfil do turista, obrigaram, de certa forma, os estabelecimentos de hospedagem de Florianópolis a adotar posturas mais prospectoras, buscando novos mercados, sobretudo o interno, oferecendo novos produtos e serviços, investindo em melhores estruturas, em treinamento de seu pessoal, em propaganda e publicidade, enfim, realizando melhorias a custos baixos para, dessa forma, fazerem frente à concorrência dos novos entrantes.

Através da análise dos resultados, pode-se contemplar o objetivo deste trabalho, que foi identificar o comportamento estratégico adotado pelos administradores. Levando-se em conta os dados da pesquisa e a especificidade do setor hoteleiro de Florianópolis, a adoção do comportamento estratégico prospector, em sua maioria, permite afirmar que os administradores estão mais atentos para as mudanças do mercado, procurando inovar em termos de produtos e serviços e entrar em novos mercados. Esse fato se deve, sobretudo, às mudanças que ocorreram no setor nos últimos anos. As maiores mudanças têm relação com o perfil dos clientes e com a entrada de novos concorrentes, que obrigaram os administradores de hotéis a adotar posturas de maior atenção para as oportunidades que surgem com a crescente entrada de turistas na cidade, somadas à mudança do seu perfil.

Por fim, pode-se afirmar que essa postura, específica do setor hoteleiro nos últimos cinco anos, pode ser atribuída à entrada, no mercado, das grandes redes hoteleiras e à mudança do perfil dos turistas, que eram, antes desse período, na sua maioria, estrangeiros que, com o câmbio favorável, traziam os dólares tão esperados pelos proprietários de hotéis. Somado a isso, ainda faziam a divulgação dos locais de hospedagem em seu país de origem. Se houvessem pesquisas desta natureza antes da crise que ocorreu no setor nos primeiros anos de 2000, provavelmente a característica da postura adotada pelos administradores teria sido diferente, tendendo a ser mais reativa e defensiva do que prospectora e analista. 


\section{REFERÊNCIAS BIBLIOGRÁFICAS}

ARRAGÓN-SANCHEZ, A.; SÁNCHEZ-MARÍN, G. Strategic orientation, management characteristics and performance: a study of Spanish SMEs. Journal of Small Business Management, v. 43, n. 3, p. $287-$ 308, Jul. 2005.

BOWMAN, C.; FAULKNER, D. Competitive and corporate strategy. London: Irwin, 1997.

CASTLE, N. G. Strategic groups and outcomes in nursing facilities. Health Care Management Review, v. 28, n. 3, p. 217-227, 2003.

CAVES, R. E.; PORTER, M. E. From entry barriers to mobility barriers: conjectural decisions and contrived deterrence to new competition. Quarterly Journal of Economics, v. 91, n. 2, p. 241-261, 1977.

CHANDLER, A. D. Strategy and structure: chapters in the history of the industrial interprise. Cambridge: M.I.T. Press, 1962.

CHILD, J. Organizational structure enviroment and performance: the role of strategic choice. Sociology, v. 6, p. 1-22, 1972.

CONANT, J. S.; MOKWA, M. P.; VARADARAJAN, P. R. Strategic types, distinctive marketing competencies and organizational performance: a multiple measures-based study. Strategic Management Journal, v. 11, p. 365-383, 1990.

COSTA, J.; TEARE, R. Developing na environmental scanning process in the hotel sector. International Journal of Contemporary Hospitaly Mangement, Bradford, v. 12, n. 3, p. 156-168, 2000.

CUSumano, M. A.; MARKIDES, C. C. Pensamento estratégico. Rio de Janeiro: Campus, 2002.

DESARBO, W. S. et al. Revisiting the Miles and Snow strategic framework: uncovering interrelationships between strategic types, capabilities, environmental uncertainty and firm performance. Strategic Management Journal, v. 26, p. 47-74, 2005.

DILL, W. R. Environment as an influence on managerial autonomy. Administrative Science Quarterly, v. 2, n. 4, p. 409-443, 1958.

DUNCAN, R. B. Characteristics of organizational environments and perceived environmental uncertainty. Administrative Science Quarterly, v. 17, p. 313-327, 1972.
EMERY, F.; TRIST, E. L. The causal texture of organizational environments. Human Relations, v. 18, p. 21-32, 1965.

FRISHAMMAR, J. Organizational environment revisited: a conceptual review and integration. International Studies of Management \& Organization, v. 36, n.3, p. 22-49, 2006.

GHEMAWAT, P. A estratégia e o cenário dos negócios: texto e casos. Porto Alegre: Bookman, 2000.

GIMENEZ, F. O estrategista na pequena empresa. Maringá: [do autor], 2000. 176p.

GIMENEZ, F. et al. Estratégia em pequenas empresas: uma aplicação do modelo de Miles e Snow. Revista de Administração Contemporânea, v. 3, n. 2, p. 53-74, 1999.

GRANT, R. M. Prospering in Dynamic-Competitive Environments: organizational capabily as know ledg integrations. Stategic Management Journal, v. 13, n. 4, p. 335-387, 1996.

GUPTA, A. K.; GOVINDARAJAN, V. An emperical examination of linkages between strategy, managerial characteristics and performance. Academy of Management Proceedings, v. 4, n. 2, p. 31-36, 1982.

GURKOV, I. Dominant strategic archetype of the Russian industrial firm. Journal for East European Management Studies, v. 12, n. 1, p. 35-53, 2007.

HAIR JR, J. F. et al. Análise multivariada de dados. Porto Alegre. Bookman, 2005.

HAMBRICK, D. C. Some tests of the effectiveness and functional attributes of Miles and Snow's Strategic Types. Academy Of Management Journal, v. 26, n. 1, p. 5-26, 1983.

. Taxonomic approaches to studying strategy: some conceptual and methodological issues. Journal of Management, v. 10, n. 1, p. 27-41, 1984.

. On the straying power of defenders, analyzers, and prospectors. Academy of Management Executive, v. 17, n. 4, p. 115-118, 2003.

HANNAN, M.; FREEMAN, J. The population ecology of organizations. American Journal of Sociology, v. 82, n. 5, p. 929-964, 1977. 
JABNOUN, N.; KHALIFAH, A.; YUSUF, A. Environmental uncertainty, strategic orientation, and quality management: a contingency model. The Quality Management Journal, v. 10, n. 4, p. 17, 2003.

JAVIDAN, M.; MURRAY, A.; RESCTENTHALER, G. Strategic change: the search for new equilibria. Journal of Organization Change Management, v. 3, n. 3, p. 59-76, 1990.

KATZ, D.; KAHN, R. L. Psicologia social das organizações. 3. ed. São Paulo: Atlas, 1987.

LAWRENCE, P.; LORCH, J. Organization and environment. Cambridge: Harvard Business School Press, 1973.

MACHADO-DA-SILVA, C. L.; FONSECA, V. S. Homogeneização e diversidade organizacional: uma visão integrativa. In: ENCONTRO ANUAL DA ANPAD, 17., 1993, Salvador. Anais... Salvador: ANPAD, 1993. v. 9, p. 147-159.

MALHOTRA, N.; FRAUZE N. Pesquisa de marketing: uma orientação aplicada. Porto Alegre: Bookman, 2001.

MILES, R. E.; SNOW, C. C. Organizational strategy, structure and process. New York: McGraw-Hill, 1978.

MILLER, A.; DESS, G. G. Assessing porter's (1980). Model in terms of its generalizability, accuracy and simplicity. Journal of Management Studies, v. 30. n. 4, p. 553-585, 1993.

MILLIKEN, F. J. Three types of perceveid uncertainty about the enverinment: state, effect and responce uncertainty. Academy of Management Review, v. 12, n. 1, p. 133-143, 1987.

MINTZBERG, H.; AHLSTRAND, B.; LAMPEL, J. Safari de estratégia: um roteiro pela selva do planejamento. Porto Alegre: Bookman, 2000.

MINTZBERG, H. I. Criando organizações eficazes: estruturas em cinco configurações. Tradução de Ciro Bernardes. São Paulo: Atlas, 1995.

OLSON, E. M.; SLATER, S. F.; HULT, G. T. M. The performance implications of fit among busines strategic, marketing organization structure and strategic Behavior. Journal of Marketing, v. 69, n. 3, p. 49-65, 2005.

PFEFFER, J.; SALANCIK, K. G. R. The external control of organizations: a resource dependence perspective. New York: Harper and Row, 1978.

POWELL, Walter; DIMAGGIO, Paul (Ed.). The new institutionalism in organizational analysis. Chicago: University of Chicago Press. 1991.

REIS, E. Estatística multivariada aplicada. Lisboa: Silabo, 1997.

ROSSETTO, C. R. Adaptação estratégica organizacional: um estudo multi-caso na indústria da construção civil - setor de edificações. 1998. $189 f$. Tese (Doutorado em Engenharia da Produção)-Universidade Federal de Santa Catarina, Florianópolis, 1998.

SLATER, S. F.; OLSON, E. M. Marketing's contribution to the implementation of business strategy: an empirical analysis. Strategic Mangement Journal, Chichester, v. 22, n. 11, p. 1055-1068, 2001.

SOUGATA, R. Environment-Strategy-Performance Linkages: a study of indian firms during economic liberalization. The Journal for Decision Makers, v. 29, n. 2, p. 9-23, 2004.

TAN, J.; TAN, D. Environment-Strategy Co-Evolution and Co-Alignment: a staged model of chinese. Strategic Management Journal, v. 26, p. 141-157, 2005.

ZAHRA, S. A.; PEARCE II, J. A. Research evidence on the Miles and Snow typology. Journal of Management, v. 16, n. 4, p. 751-768, 1990.

ZUCKER, L. G. Normal change or risk business: institucional effects on the "hazard" of change in hospital organizations, 1959-1979. Journal of Management Studies, v. 24, n. 6, p. 671-700, 1987.

ZÚÑIGA-VICENTE, J. Á.; VICENTE-LORENTE, J. D. Strategic moves and organizational survival in turbulent environments: the case of Spanish Banks (1983-1997). Journal of Management Studies, v. 43, n. 3, p. 484-519, May 2006 